\title{
Spectral Solution of Time-Dependent Shallow Water Hydroelasticity
}

\author{
Michael H. Meylan \\ Institute of Information and Mathematical Sciences, Massey University New Zealand
}

\section{Introduction}

If a hydroelastic problem is linear the time-dependent motion can be found using spectral theory, at least theoretically. However the spectral theory for linear hydroelasticity has not been developed, even for the simplest cases. For example, the work of [1] which presented a method to determine the scattering frequencies for hydroelastic problems did not develop any spectral theory. This has meant that spectral methods to solve the time-dependent motion, such as [4], have only solved for the motion is restrictive cases.

In this paper the spectral theory for a simple hydroelastic problem, the two dimensional thin plate floating on shallow water, is developed. This work, while it has practical applications, primarily aims to motivate the development of a spectral theory for more complicated hydroelastic problems. Two separate methods are developed, both based on a special inner product which represents the energy of the plate-water system. The first method uses the single frequency solutions as the eigenfunctions and the problem is solved by a generalised Fourier transform. The second method is based on Lax-Philips scattering and calculates the solution by an expansion in modes. These modes represent the natural frequencies of the plate-water system and include the decay due to the radiation of energy into the surrounding water.

\section{Formulation: A Thin Plate on Shallow Water}

A thin plate of shallow draft covers the region $-b \leq x \leq b$ of shallow water of depth $h$. The mathematical description of the problem follows from [5]. The kinematic condition is

$$
\partial_{t} \zeta=-h \partial_{x}^{2} \phi
$$

where $\phi$ is the velocity potential of the water and $\zeta$ is the displacement of the water surface or the plate. The equation for the pressure is

$$
-\rho g \zeta-\rho \partial_{t} \phi=\left\{\begin{array}{c}
0, \quad x \notin(-b, b), \\
D \partial_{x}^{4} \zeta+\rho^{\prime} d \partial_{t}^{2} \zeta, \quad x \in(-b, b),
\end{array}\right.
$$

where $D$ is the bending rigidity of the plate per unit length, $\rho$ is the density of water, $\rho^{\prime}$ is the density of the plate, $g$ is the acceleration due to gravity, and $d$ is the thickness of the plate. We also have the free edge boundary conditions

$$
\lim _{x \downarrow-b} \partial_{x}^{2} \zeta=\lim _{x \uparrow b} \partial_{x}^{2} \zeta=\lim _{x \downarrow-b} \partial_{x}^{3} \zeta=\lim _{x \uparrow b} \partial_{x}^{3} \zeta=0 .
$$

Non-dimensional variables are now introduced. The space variables are non-dimensionalised using the water depth $h$, and the time variables are non dimensionalised using $\sqrt{\frac{h}{g}}$. The non-dimensionalised variables are

$$
\bar{x}=\frac{x}{h}, \bar{t}=t \sqrt{\frac{g}{h}}, \bar{\zeta}=\frac{\zeta}{h}, \text { and } \bar{\phi}=\frac{\phi}{h^{2} \sqrt{g / h}} .
$$

In these new variables, ([2.1]) and ([2.2]) become

$$
\partial_{\bar{t}} \bar{\zeta}=-\partial_{\bar{x}}^{2} \bar{\phi}
$$

and

subject to the boundary conditions

$$
-\bar{\zeta}-\partial_{\bar{t}} \bar{\phi}=\left\{\begin{array}{c}
0, \bar{x} \notin(-\bar{b}, \bar{b}), \\
\beta \partial_{\bar{x}}^{4} \bar{\zeta}+\gamma \partial_{\bar{t}}^{2} \bar{\zeta}, \quad x \in(-\bar{b}, \bar{b}),
\end{array}\right.
$$

$$
\lim _{\bar{x} \downarrow-\bar{b}} \partial_{\bar{x}}^{2} \bar{\zeta}=\lim _{\bar{x} \uparrow \bar{b}} \partial_{\bar{x}}^{2} \bar{\zeta}=\lim _{\bar{x} \downarrow-\bar{b}} \partial_{\bar{x}}^{3} \bar{\zeta}=\lim _{\bar{x} \uparrow \bar{b}} \partial_{\bar{x}}^{3} \bar{\zeta}=0 .
$$

The non-dimensional variables $\beta$ and $\gamma$ are given by

$$
\beta=\frac{D}{\rho g h^{4}} \text { and } \gamma=\frac{\rho^{\prime} d}{\rho h} .
$$

For clarity the overbar is dropped from now on. Following [3] we set the inertia term, $\gamma \partial_{t}^{2} \zeta$ to zero since it is much 
smaller than $\zeta$. This follows from the fact that $d \ll h$ and, since the water is shallow, the wavelengths (and hence the frequencies) must be much greater than $h$ so that $\partial_{t}^{2} \zeta \ll \zeta$.

\section{The self-adjoint solution method}

In this section, a solution for the time dependent motion of the plate-water system is developed using the theory of self-adjoint operators. We define a two-component variable $U(x, t)$ by

$$
U(x, t)=\left(\begin{array}{c}
\phi(x, t) \\
i \zeta(x, t)
\end{array}\right)
$$

Equations ([2.4]) and ([2.5]) are now converted to the following equation for $U$

$$
\frac{1}{i} \partial_{t} U=\mathcal{P} U
$$

where

$$
\mathcal{P}=\left(\begin{array}{cc}
0 & 1+\beta(H(x-b)-H(x+b)) \partial_{x}^{4} \\
-\partial_{x}^{2} & 0
\end{array}\right)
$$

and $H$ is the Heavyside function. Equation ([3.2]) is also subject to the boundary conditions at the end of the plate given by equation ([2.6]) and the initial condition

$$
U(x, t)_{t=0}=U_{0}(x)=\left(\begin{array}{c}
\phi_{0}(x) \\
i \zeta_{0}(x)
\end{array}\right)
$$

The operator $\mathcal{P}$ is self adjoint in the Hilbert space $\mathcal{H}$ with inner product given by the energy. The energy inner product for the two vectors

is

$$
U_{1}=\left(\begin{array}{c}
\phi_{1} \\
i \zeta_{1}
\end{array}\right) \text { and } U_{2}=\left(\begin{array}{c}
\phi_{2} \\
i \zeta_{2}
\end{array}\right)
$$

$$
\left\langle U_{1}, U_{2}\right\rangle_{\mathcal{H}}=\left\langle\partial_{x} \phi_{1}, \partial_{x} \phi_{2}\right\rangle+\left\langle\left(1+\beta(H(x-b)-H(x+b)) \partial_{x}^{4}\right) i \zeta_{1}, i \zeta_{2}\right\rangle .
$$

The subscript $\mathcal{H}$ is used to denote the special inner product and the angle brackets without the $\mathcal{H}$ denote the standard inner product

$$
\langle f(x), g(x)\rangle=\int_{-\infty}^{\infty} f(x) g^{*}(x) d x .
$$

Since $\mathcal{P}$ is self-adjoint the solution to ([3.2]) can be calculated using the eigenfunctions of $\mathcal{P}$. There are two eigenfunctions for each eigenvalue $\lambda \in \mathbb{R}$, a unit incoming wave from the left $(x=-\infty)$ denoted by $\Phi^{>}$and a unit wave incoming from the right $(x=\infty)$ denoted by $\Phi^{<}$. The eigenfunctions $\Phi^{>}(\lambda, x)$ consist of the two components $\phi^{>}(\lambda, x)$ and $i \zeta^{>}(\lambda, x)$ given by

and

$$
\phi^{>}(\lambda, x)=\left\{\begin{array}{c}
e^{-i \lambda x}+S_{11}(\lambda) e^{i \lambda x}, x<-b, \\
\sum_{j=1}^{6} \alpha_{j} e^{\mu_{j}(\lambda) x}, \quad-b<x<b \\
S_{12}(\lambda) e^{-i \lambda x}, \quad x>b
\end{array}\right.
$$

where $\mu_{j}(\lambda)$ are the six roots of the equation

$$
i \zeta^{>}(\lambda, x)=\left\{\begin{array}{c}
\lambda e^{-i \lambda x}+\lambda S_{11}(\lambda) e^{i \lambda x}, \quad x<-b, \\
\frac{-1}{\lambda} \sum_{j=1}^{6} \mu_{j}(\lambda)^{2} \alpha_{j} e^{\mu_{j}(\lambda) x}, \quad-b<x<b \\
\lambda S_{12}(\lambda) e^{-i \lambda x}, \quad x>b,
\end{array}\right.
$$

$$
\beta \mu^{6}+\mu^{2}+\lambda^{2}=0
$$

and the values of $S_{11}(\lambda), S_{12}(\lambda)$, and $\alpha_{j}$ are chosen so that $\phi^{>}(\lambda, x)$ and $\zeta^{>}(\lambda, x)$ satisfy the boundary conditions ([2.3]) and the continuity of $\phi$ and $\partial_{x} \phi$ at $x= \pm b$. Also, since $S_{11}$ represents the amplitude of the reflected wave and $S_{12}$ represents the amplitude of the transmitted wave, conservation of energy requires that $\left|S_{11}\right|^{2}+\left|S_{12}\right|^{2}=1$. The eigenfunctions for the wave propagating from the right $\Phi^{<}$are found similarly.

Since the boundary conditions are symmetric we must have $S_{22}(\lambda)=S_{11}(\lambda)$ and $S_{12}(\lambda)=S_{21}(\lambda)$. Also, the scattering matrix

$$
\mathbf{S}(\lambda)=\left(\begin{array}{ll}
S_{11}(\lambda) & S_{12}(\lambda) \\
S_{21}(\lambda) & S_{22}(\lambda)
\end{array}\right)
$$

is unitary as a consequence of the Lax-Philips scattering structure which will be discussed in section 4.

Equation ([3.2]) can be solved by a generalised Fourier transform based on the eigenfunctions of the operator $\mathcal{P}$. The eigenfunctions are orthogonal since $\mathcal{P}$ is self-adjoint and the normalising constant is determined by calculating the inner 
product of these eigenfunctions with themselves. These inner products are given by

and

$$
\begin{aligned}
\left\langle\Phi^{>}\left(x, \lambda_{1}\right), \Phi^{>}\left(x, \lambda_{2}\right)\right\rangle_{\mathcal{H}} & =4 \pi \delta\left(\lambda_{1}-\lambda_{2}\right) \lambda_{1}^{2}, \\
\left\langle\Phi^{<}\left(x, \lambda_{1}\right), \Phi^{<}\left(x, \lambda_{2}\right)\right\rangle_{\mathcal{H}} & =4 \pi \delta\left(\lambda_{1}-\lambda_{2}\right) \lambda_{1}^{2},
\end{aligned}
$$

Using these eigenfunctions the solution to ([3.2]) subject to ([3.3]) is

$$
\left\langle\Phi^{>}\left(x, \lambda_{1}\right), \Phi^{<}\left(x, \lambda_{2}\right)\right\rangle_{\mathcal{H}}=0 .
$$

$$
\begin{aligned}
U(x, t)= & \int_{-\infty}^{\infty}\left\langle U_{0}(x), \frac{\Phi^{>}(x, \lambda)}{4 \pi \lambda^{2}}\right\rangle_{\mathcal{H}} \Phi^{>}(x, \lambda) e^{i \lambda t} d \lambda \\
& +\int_{-\infty}^{\infty}\left\langle U_{0}(x), \frac{\Phi^{<}(x, \lambda)}{4 \pi \lambda^{2}}\right\rangle_{\mathcal{H}} \Phi^{<}(x, \lambda) e^{i \lambda t} d \lambda .
\end{aligned}
$$

\section{The Lax-Philips Scattering Solution Method}

In this section, a solution to the time-dependent motion of the plate-water system is developed using the Lax-Philips scattering theory ([2]). This solution method will only solve for an initial condition which is zero outside the region of water covered by the plate $(|x|>b)$. The basic idea and consequences of the Lax-Philips scattering theory will be outlined here for our specific problem. The Hilbert space $\mathcal{H}$ is decomposed into three subspaces called the incoming, outgoing and scattering spaces. The incoming space, denoted by $D_{-}$, consists of all waves travelling towards the plate, either from the left or the right, as appropriate. The outgoing subspace, denoted by $D_{+}$, consists of all waves travelling away from the plate, either to the left or right, as appropriate. What remains is called the scattering region, and is denoted by $K$. In our problem, $K$ is nothing more than the potential and displacement under the plate.

We introduce a new operator $\mathcal{B}$ which describes the evolution of the plate in the absence of wave forcing. If we again denote the motion of the plate by the two component vector $U(x, t)$ ([3.1]) then the equation for the motion of the plate in the absence of wave forcing is

$$
\frac{1}{i} \frac{\partial}{\partial t} U=\mathcal{B} U
$$

This means that $\mathcal{B}$ is the infinitesimal generator of the semigroup (dissipative evolution operator) given by restricting the problem to $K$, i.e.,

$$
e^{i \mathcal{B} t}=\left.P_{K} e^{i \mathcal{P} t}\right|_{K}
$$

where $P_{K}$ is the projection onto the subspace $K$ and the $\left.\right|_{K}$ means that the input is restricted to $K$. Therefore $e^{i \mathcal{B} t}$ is the evolution of an initial condition which is zero outside $K$ and which is subsequently restricted to $K$, i.e. the evolution of the plate motion in the absence of wave forcing.

The solution to the non self-adjoint problem requires the eigenvalues and eigenfunctions of $\mathcal{B}$, sometimes referred to as scattering frequencies or resonances. The eigenvalues of $\mathcal{B}$ are found using the analytic extension of the scattering matrix $\mathbf{S}(\lambda)$ ([3.8]) to $\mathbb{C}$ since the eigenvalues of $\mathcal{B}$ occur precisely at the singularities of $\mathbf{S}(\lambda)$. These singularities are found by a complex integration search method to give a rough estimate and Newton's method to determine their location accurately.

The eigenfunctions of $\mathcal{B}$ associated with the eigenvalue $\lambda_{n}$ are denoted by $\Phi^{+}\left(\lambda_{n}, x\right)$, and those of $\mathcal{B}^{*}$ (the adjoint of $\mathcal{B})$ associated with the eigenvalue $\lambda_{n}^{*}$ are denoted by $\hat{\Phi}^{+}\left(\lambda_{n}^{*}, x\right)$. That is,

$$
\mathcal{B} \Phi^{+}\left(\lambda_{n}, x\right)=\lambda_{n} \Phi^{+}\left(\lambda_{n}, x\right)
$$

and

The eigenfunction $\Phi^{+}\left(\lambda_{n}, x\right)$ is given by

$$
\mathcal{B}^{*} \hat{\Phi}^{+}\left(\lambda_{n}^{*}, x\right)=\lambda_{n}^{*} \hat{\Phi}^{+}\left(\lambda_{n}^{*}, x\right) .
$$

$$
\Phi^{+}\left(\lambda_{n}, x\right)=\left(\begin{array}{c}
\phi^{+}\left(\lambda_{n}, x\right) \\
i \zeta^{+}\left(\lambda_{n}, x\right)
\end{array}\right)=\left(\begin{array}{c}
\sum_{j=1}^{6} \alpha_{j} e^{\mu_{j}\left(\lambda_{n}\right) x} \\
6-\frac{\alpha_{j} \mu_{j}\left(\lambda_{n}\right)^{2}}{\lambda_{n}} e^{\mu_{j}\left(\lambda_{n}\right) x}
\end{array}\right)
$$

where $\mu_{j}(\lambda)$ are the six roots of equation ([3.7])

$$
\beta \mu_{j}(\lambda)^{6}+\mu_{j}(\lambda)^{2}+\lambda^{2}=0 .
$$

The $\alpha_{j}$ are determined by the condition that the waves are of unit amplitude and are outgoing at $x=-b$,

$$
\phi^{+}\left(\lambda_{n},-b\right)=e^{i \lambda_{n} b},\left.\quad \partial_{x} \phi^{+}\left(\lambda_{n}, x\right)\right|_{x=-b}=i \lambda_{n} e^{i \lambda_{n} b},
$$


and the boundary conditions at the end of the plate ([2.3]) are satisfied. The eigenfunctions of $\mathcal{B}^{*}$ are found similarly.

A biorthogonal system with respect to the energy inner product ([3.4]) is formed by the eigenfunctions of $\mathcal{B}, \Phi^{+}\left(\lambda_{n}, x\right)$, and the eigenfunctions of $\mathcal{B}^{*}, \hat{\Phi}^{+}\left(\lambda_{n}, x\right)$. To normalise the biorthogonal system, the inner product of $\Phi^{+}\left(\lambda_{n}, x\right)$ and $\hat{\Phi}^{+}\left(\lambda_{n}, x\right)$ must be found and this can be calculated analytically. Once this has been accomplished the evolution of the plate from some initial displacement $U_{0}(x)$ is given by

$$
U(x, t)=\sum_{n=-\infty}^{\infty} e^{i \lambda_{n} t} \frac{\left\langle U_{0}(x), \hat{\Phi}\left(\lambda_{n}, x\right)\right\rangle_{\mathcal{H}}}{\left\langle\Phi\left(\lambda_{n}, x\right), \hat{\Phi}\left(\lambda_{n}, x\right)\right\rangle_{\mathcal{H}}} \Phi\left(\lambda_{n}, x\right)
$$

where $U_{0}(x)$ is the initial condition given by equation ([3.3]).

\section{Summary}

Two methods have been presented to solve the time dependent motion of a thin plate floating on shallow water. One method was based on self-adjoint operator theory, and the other on Lax-Philips scattering. The Lax-Philips method only solved the problem of a free plate and cannot be used to solve for wave forcing. The self-adjoint method solves both the wave forcing and free plate problem. The eigenfunctions for the self-adjoint method are orthogonal and the eigenvalues are continuous and consist of all $\mathbb{R}$, which makes the calculation of the eigenvalues trivial. The Lax-Philips method has discrete eigenvalues and the system of eigenfunctions is biorthogonal. The eigenvalues for the Lax-Philips method must be calculated numerically. however the Lax-Philips method has the significant advantage that the modes of vibration of the plate-water system and their frequency and rate of decay are found.

\section{References}

[1] C. Hazard and M. Lenoir. Determination of scattering frequencies for an elastic floating body. SIAM J. Math. Anal., 24(4):1458-1514, 1993.

[2] P. D. Lax and R. S. Philips. Scattering Theory. Academic, 1989.

[3] Y. Namba and M. Ohkusu. Hydroelastic behaviour of floating artifical islands in waves. Int. J. of Offshore and Polar Engineering, 9(2):39-47, 1999.

[4] S. Ohmatsu. Numerical calculation of hydroelastic behavior of VLFS in time domain. In M. Kashiwagi, W. Koterayama, and M. Ohkusu, editors, Hydroelasticity in Marine Technology, pages 89-97. Yomei Printing Cooperative Society, Fukuoka, Japan, 1998.

[5] J. J. Stoker. Water Waves: The Mathematical Theory with Applications. Interscience, 1957. 